\title{
On the Use of Perturbation Theory in Eigenvalue Problems
}

\author{
Arij A. Awin, Bashir W. Sharif, Ali M. Awin \\ Department of Mathematics, Faculty of Science, University of Tripoli, Tripoli, Libya \\ Email: awinsus@yahoo.com
}

How to cite this paper: Awin, A.A., Sharif, B.W. and Awin, A.M. (2021) On the Use of Perturbation Theory in Eigenvalue Problems. Journal of Applied Mathematics and Physics, 9, 2224-2243.

https://doi.org/10.4236/jamp.2021.99142

Received: August 5, 2021

Accepted: September 12, 2021

Published: September 15, 2021

Copyright (c) 2021 by author(s) and Scientific Research Publishing Inc. This work is licensed under the Creative Commons Attribution International License (CC BY 4.0).

http://creativecommons.org/licenses/by/4.0/ (c) (i) Open Access

\begin{abstract}
The importance of perturbation theory in many fields is very clear through almost a century or even more. Its importance was exemplified in solving many problems in physics and other applied fields. A great deal of applications arose in dealing with eigenvalue problems especially in quantum mechanics in conjunction with the field of atomic physics. Accordingly, it came to our mind to write a brief review article on the subject. At the beginning, we give some important definitions to do with various eigenvalue problems; then we introduce concepts that have to do with perturbation theory and the techniques used in such a theory, beginning with the algebraic perturbation theory giving a good number of examples from the literature on the use of the theory in solving integral equation, algebraic equations and differential equations. Few applications are then given in applied fields such as classical mechanics, quantum mechanics and fluid mechanics. Finally, a concluding discussion is given which is related to the use of the theory.
\end{abstract}

\section{Keywords}

Perturbation, Eigenvalue, Hamiltonian, Quantum, Mechanics

\section{Introduction}

The simplest eigenvalue problem in Algebra is that of finding the value of $\lambda$ which makes the square matrix $A$ diagonal, and this is solved by writing the characteristic equation which is

$$
\operatorname{det}(A-\lambda I)=0
$$

And solving for its roots, $I$ is the unit matrix. However, the problem becomes more interesting when dealing with symmetric matrices since those are of importance in applied fields such as in sound in determining natural modes of vi- 
brations or in elasticity. Such problems will stimulate using different computer software such as subroutine JACOBI [1].

Another kind of eigenvalue problems is that encountered in integral equations, e.g. to solve the eigenvalue problem [2]

$$
f_{n}(x)=\lambda_{n} \int_{a}^{b} \mathrm{~d} y K(x, y) f_{n}(y)
$$

Most important are the eigenvalue problems, faced with, in quantum mechanics, i.e. when the time-dependent Schrodinger equation or the time-independent one are under consideration and the energy values are required for a certain system (e.g. the energy levels of the Hydrogen atom). The time-independent Schrodinger equation, as an eigenvalue problem, is written as

$$
\boldsymbol{H} \varphi=E \varphi
$$

where $\boldsymbol{H}$ is the Hamiltonian, $E$ is the energy eigenvalue, and $\varphi$ is the eigenfunction. Note that $\boldsymbol{H}$ is given by

$$
H=-\frac{\hbar^{2}}{2 m} \nabla^{2}+V(\vec{r})
$$

$\nabla^{2}$ is the Laplacian.

It is to be noted that dealing with eigenvalue problems, the operators encountered in the calculation are of the Hermitian type and that the Hamiltonian is Hermitian [3].

Keeping in mind the above small piece of information about the eigenvalue problems, we now turn to answer the important question: What is perturbation theory?

Perturbation theory is a theory built on certain mathematical methods used in order to get an approximate solution to some given problem with the condition that we start with an exact known solution for the related simpler problem. This means that the problem is partitioned into two parts: one is solvable and the other part represents the perturbation. Hence, perturbation theory is only used when the original problem cannot be solved exactly, but it can be formulated through the addition of a small perturbation term to the problem which can be solved exactly.

The ultimate solution to the original problem is obtained as a series called perturbation series. The first term of which is the exact solution of the related problem (without perturbation) and the other terms give the deviations in the exact solution; in fact, this gives us the approximate solution to the problem. It is clear that the more terms we take from the series, the better result we get.

In principle, the preliminary steps of performing perturbation techniques are as follows:

Let $\epsilon$ be a small parameter and $S$ is a certain solution to be approximated in the problem, then we arrive to a series of the type:

$$
S=S_{0}+\epsilon^{1} S_{1}+\epsilon^{2} S_{2}+\cdots=\sum_{i=0}^{\infty} S_{i} \epsilon^{i}
$$

where $S_{0}$ is the solution to the unperturbed problem and the approximation in 
the original problem starts with the term $\epsilon^{1} S_{1}$. Hence, we get the approximate solution for the problem by truncating the above series; e.g. the approximate solution of the first order is

$$
S \cong S_{0}+\epsilon^{1} S_{1}
$$

In a way, perturbation theory is similar to the methods used in numerical analysis. The first early time, the theory was used was in space mechanics to evaluate the moon's orbit; however, perturbation theory is now widely used in many fields, such as wave mechanics, statistical mechanics, quantum mechanics, and chemistry [4].

In quantum mechanics, perturbation theory is used to get approximate solutions for complex quantum systems in terms of simpler ones. The idea is to start with a simple system which is solvable then to add a weak perturbation Hamiltonian, and if the perturbation is small then the different physical phenomena (observables) which are connected with the perturbed system, such as energy levels and eingenfunctions, can be expressed as corrections to the corresponding simple system. Due to the smallness of these corrections they can be calculated via approximate methods in the form of converging series.

Perturbation theory is a good and important tool to describe real quantum systems noting that the exact solutions of Schrodinger equation for some Hamiltonians are difficult to obtain even if these Hamiltonians are not that complex [5].

In the next section, we give some examples on the use of perturbation theory in solving algebraic equations and in solving differential and integral equations. In Section 3, we direct our attention to its uses in solving eigenvalue problems in many applied fields. Finally we conclude shedding light on some recent uses of perturbation theory.

\section{Algebraic Perturbation Theory}

As mentioned, earlier, one of the important eigenvalue problems are of the type of Equation (2), i.e. it is an integral equation and where $K(x, y)$ is the kernel; if this kernel is separable, which means that:

$$
K(x, y)=U(x) V(y)
$$

Then, one can get non-trivial solutions (eigenvalues and eigenfunctions).

Taking into account Equation (2) and Equation (7), we see that

$$
f_{n}(x)=\lambda_{n} A U(x), A \text { is a constant }
$$

Substituting, the eigenvalue will be given by

$$
\lambda_{n}=\frac{1}{\int_{a}^{b} U(y) V(y) \mathrm{d} y}
$$

Now if $U(y)=V(y)=\sin y$, then $f(x)=\lambda A \sin x$ and the eigenvalue is given by $\lambda=\frac{4}{\pi}[2]$. 


\subsection{Small Perturbation Parameter}

If we consider the eigenvalue problem:

$$
\int_{-\infty}^{\infty} \mathrm{e}^{-|x|-|y|} U(y) \mathrm{d} y=\lambda U(x),-\infty<x<\infty
$$

And if we are looking for a value of $\lambda$ giving a solution $U(x) \neq 0$ for this equation fulfilling the condition that $\int_{-\infty}^{\infty}|U(x)|^{2}<\infty$; then $U(x)$ is an eigenfunction corresponding to the eigenvalue $\lambda$. In this case the spectrum of the eigenvalues is discrete.

For the eigenvalue $\lambda$, it is clear, with a straight forward manipulation that $\lambda=1$ and the corresponding eigenfunction is $\varphi_{0}=\mathrm{e}^{-|x|} \quad[6]$.

Now, consider the following eigenvalue problem

$$
\int_{-\infty}^{\infty} \mathrm{e}^{-|x|-|y|} U(y) \mathrm{d} y+\epsilon x U(x)=\lambda U(x),-\infty<x<\infty
$$

where $\epsilon$ is a given real number. If $\epsilon$ is assumed to be of small value, then the problem in this case is to find the eigenvalue $\lambda=\lambda(\epsilon)$ and the corresponding eigenfunction $U=U(x, \epsilon)$. To solve the problem, we write the two following series

$$
\lambda=\lambda_{0}+\epsilon \lambda_{1}+\epsilon^{2} \lambda_{2}+\cdots ; \lambda_{0}=1
$$

And

$$
U=\varphi_{0}+\epsilon \varphi_{1}+\epsilon^{2} \varphi_{2}+\cdots ; \varphi_{0}=\mathrm{e}^{-|x|}
$$

Moreover we put

$$
A U \equiv \int_{-\infty}^{\infty} \mathrm{e}^{-|x|-|y|} U(y) \mathrm{d} y ; B U \equiv x U(x)
$$

So as to get

$$
(A+\epsilon B)\left(\varphi_{0}+\epsilon \varphi_{1}+\epsilon^{2} \varphi_{2}+\cdots\right)=\left(\lambda_{0}+\epsilon \lambda_{1}+\epsilon^{2} \lambda_{2}+\cdots\right)\left(\varphi_{0}+\epsilon \varphi_{1}+\epsilon^{2} \varphi_{2}+\cdots\right)
$$

And if we assume that the series $\varphi_{0}+\epsilon \varphi_{1}+\epsilon^{2} \varphi_{2}+\cdots$ is convergent in order to be able to perform multiplication term by term, then on comparing coefficients of $\epsilon^{i}$ we get

$$
A \varphi_{0}-\lambda_{0} \varphi_{0}=0 ; A \varphi_{1}-\lambda_{0} \varphi_{1}=\lambda_{1} \varphi_{0}-B \varphi_{0}
$$

In general for the nth terms, one gets

$$
A \varphi_{n}-\lambda_{0} \varphi_{n}=\lambda_{n} \varphi_{0}+\lambda_{n-1} \varphi_{1}+\cdots+\lambda_{1} \varphi_{n-1}-B \varphi_{n-1}
$$

From Equation (16) we see that $\lambda=1$ and $\varphi_{0}=\mathrm{e}^{-|x|}$, as expected.

The last equation is written in the form

$$
A \varphi_{n}-\lambda_{0} \varphi_{n}=f
$$

and where

$$
f \equiv \lambda_{n} \varphi_{0}+\lambda_{n-1} \varphi_{1}+\cdots+\lambda_{1} \varphi_{n-1}-B \varphi_{n-1}
$$

Taking the inner product of Equation (18) with $\varphi_{0}$ and noting that the kernel is symmetric in $x$ and $y$, one gets

$$
\lambda_{1}=\left(\varphi_{0}, B \varphi_{0}\right)
$$


$\lambda_{1}$ is the first order correction to the eigenvalue. Equation (20) can be stated as "The approximation of first order in the eigenvalue is equal to the mean value of the perturbation operator $B$ with respect to the normalized unperturbed eigenfunction $\varphi_{0} "[6][7]$.

In this problem $\lambda_{1}=\int_{-\infty}^{\infty} x \mathrm{e}^{-2|x|} \mathrm{d} x=0$. Moreover, the correction to the eigenfunction $\varphi_{1}$ is given by $\varphi_{1}=x \mathrm{e}^{-|x|}$.

Calculating $\lambda_{2}$ and $\varphi_{2}$ in the same manner, one obtains $\lambda$ and $U$ as

$$
\lambda=1+\frac{1}{2} \epsilon^{2}+\ldots
$$

And

$$
U=\mathrm{e}^{-|x|}\left(1+x \epsilon+\left(x^{2}-\frac{3}{4}\right) \epsilon^{2}+\cdots\right)
$$

\subsection{Algebraic Equations}

To illustrate the use of perturbation theory in solving certain algebraic equations, we give the following examples:

\section{Example 1}

Consider the cubic equation $x^{3}-x+\epsilon=0$, where $\epsilon$ is a small perturbation number and assume that the solution is of the form

$$
x=x_{0}+\epsilon x_{1}+\epsilon^{2} x_{2}+O\left(\epsilon^{3}\right)
$$

where $O\left(\epsilon^{3}\right)$ is a term containing all terms with $\epsilon^{3}$ and higher. Substituting in the cubic equation and equating coefficients of $\epsilon^{i}$, we get

$$
x_{0}^{3}-x_{0}=0,3 x_{0}^{2} x_{1}-x_{1}+1=0,3 x_{0} x_{2}-x_{2}+3 x_{0} x_{1}^{2}=0
$$

From which, we obtain $x_{0}=0, \pm 1$ and $x_{1}=\frac{1}{1-3 x_{0}^{2}}$. Therefore, for $x_{0}=0$ we get $x_{1}=1$ and $x=\epsilon+O\left(\epsilon^{2}\right)$; while for $x_{0}= \pm 1$ we get $x= \pm 1-\frac{1}{2} \epsilon+O\left(\epsilon^{2}\right)$.

Continuing this process we can have the solution as convergent expansion in $\epsilon$. The truncation in the series is determined by the degree of precision we like to reach. Moreover, the same result can be obtained using the usual Taylor expansion [7].

\section{Example 2}

Again, we consider the cubic equation $\epsilon x^{3}-x+1=0$; using this with the familiar expansion in $\epsilon$ and on substitution, we get $-x_{0}+1=0,-x_{1}+x_{0}^{3}=0$, $-x_{2}+3 x_{1} x_{0}^{2}=0$; for $x_{0}=x_{1}=1$, we have $x(\epsilon)=1+\epsilon+O\left(\epsilon^{2}\right)$, this gives the linear part of the solution. As to the two other solutions, we note that as $\epsilon \rightarrow 0$, $x(\epsilon) \rightarrow \infty$. This is actually a standard singular perturbation problem. To overcome this problem, some sort of scaling is needed; this is done by writing $x$ as $x(\epsilon)=\frac{1}{\rho(\epsilon)} y(\epsilon)$ so as $y=O(1)$ as $\epsilon \rightarrow 0$, and from the cubic equation one gets $\frac{\epsilon y^{3}}{\rho^{3}}-\frac{y}{\rho}+1=0 . \rho$ needs to be determined in order to fulfill our 
requirement, namely to get a definite answer. For that, the principle of dominant balance is to be used; this requires that the two leading terms in the equation, at least, should have the same order of magnitude. Hence $\frac{\epsilon}{\rho^{3}}=\frac{1}{\rho}$ giving $\rho=\sqrt{\epsilon}$. Our cubic equation, now, becomes, in terms of the variable $y, y^{3}-y+\sqrt{\epsilon}=0$. Following the steps of the last example, we get $y= \pm 1-\frac{1}{2} \epsilon^{\frac{1}{2}}+O(\epsilon)$ or $x= \pm \frac{1}{\sqrt{\epsilon}}-\frac{1}{2}+O\left(\epsilon^{1 / 2}\right) ;$ which are the other two solutions of the problem [7].

\section{Example 3}

If the quadratic equation $(1-\epsilon) x^{2}-2 x+1=0$ is to be solved, then making the usual expansion in $\epsilon$ and comparing coefficients of $\epsilon^{i}$, we get $x_{0}^{2}-2 x_{0}+1=0$, $2\left(x_{0}-1\right) x_{1}=x_{0}^{2}$; the first equation leads to the solution $x_{0}=1$ which is a repeated root, substituting in the second equation we get a contradiction $(0=1$ ? $)$ which means that the series techniques do not work here. Hence, we calculate the roots of the equation using the simple general square root method to get $x_{ \pm}=\frac{1 \pm \sqrt{\epsilon}}{1-\epsilon}$; that gives us a hint for writing the expansion as $x=x_{0}+\sqrt{\epsilon} x_{1}+\epsilon x_{2}+O\left(\epsilon^{\frac{3}{2}}\right)$ instead of the expansion $x=x_{0}+\epsilon x_{1}+O\left(\epsilon^{2}\right)$. Now following the same techniques used before we get $x_{0}=1, x_{1}= \pm 1$ and hence the solutions are $x=1 \pm \sqrt{\epsilon}+O(\epsilon)$ as expected [7].

\section{Example 4}

Given that $x \mathrm{e}^{-x}=\epsilon$, then we see that there are two possible solutions for it for the case $\epsilon \rightarrow 0^{+}$, namely

1) When $x \rightarrow 0$ and $x=\epsilon+\epsilon^{2}+O\left(\epsilon^{3}\right)$.

2) When $\mathrm{e}^{-x} \rightarrow 0$ as $x \rightarrow \infty$, for this case $x$ has to behave like $\log (1 / \epsilon)$; and in order to get a convergent solution, we write the problem as $\mathrm{e}^{-x}=\epsilon / x$ which implies that $x=\log x+\log (1 / \epsilon)$, note that $x_{1}=\log (1 / \epsilon)$ is the initial starting point for any recurrence techniques we choose.

Using the formula $x_{n+1}=\log x_{n}+\log (1 / \epsilon), n \in N$ and defining $L_{1}=\log (1 / \epsilon)$, $L_{2}=\log (\log (1 / \epsilon))$, we see that $x_{2}=L_{1}+L_{2}, \quad x_{3}=L_{1}+\log \left(L_{1}+L_{2}\right)$, etc.

Note that the successive terms of the series converge slowly as $\epsilon \rightarrow 0$ [7].

\subsection{Examples from Different Mathematics Fields}

In this subsection, we give applications of perturbation theory in other different fields of mathematics such as differential equations and geometry. These are illustrated by few examples.

\section{Example 5}

Given the ordinary differential equation $\frac{\mathrm{d}^{2} y}{\mathrm{~d} x^{2}}=-\epsilon \frac{\mathrm{d} y}{\mathrm{~d} x}-1, \quad y(0)=0, \dot{y}(0)=1$, which represents a projectile vertical motion in air with air resistance force taken into account. $\epsilon=k v / m g$, where $v$ is the initial velocity, $m$ is the projectile mass, 
$k$ is the friction constant, and $g$ is the gravitational constant. If we want now to solve for $y$, we get the exact solution as $y(x)=\frac{1+\epsilon}{\epsilon^{2}}\left(1-\mathrm{e}^{-\epsilon x}\right)-x / \epsilon$; and if $0<\epsilon \leq 1$ which corresponds to the case when the air resistance is weak, then we use Taylor's series to obtain the first few terms of the expansion as $y(x)=x-\frac{x^{2}}{2}+\epsilon\left(-\frac{x^{2}}{2}+\frac{x^{3}}{6}\right)+\epsilon^{2}\left(\frac{x^{3}}{6}-\frac{x^{4}}{24}\right)+O\left(\epsilon^{3}\right)$. The expansion is valid for $0<x \ll 1 / \epsilon$.

Now, assuming that we are not aware of the exact solution, then we use perturbation theory to write $y(x)=y_{0}(x)+\epsilon y_{1}(x)+\epsilon^{2} y_{3}(x)+O\left(\epsilon^{3}\right)$; and where $y_{i}(x), i=0,1,2, \cdots$ are to be determined; as before, we substitute this in the original equation, following the same old techniques, keeping in mind that the initial conditions become $y_{0}(0)+\epsilon y_{1}(0)+\epsilon^{2} y_{2}(0)+O\left(\epsilon^{3}\right)=0$, $\dot{y}_{0}(0)-1+\epsilon \dot{y}_{1}+\epsilon^{2} \dot{y}_{2}+O\left(\epsilon^{3}\right)=0$; then we get the system of differential equations $\ddot{y}_{0}+1=0, y_{0}(0)=0, \dot{y}_{0}(0)=1 ; \ddot{y}_{1}+\dot{y}_{0}=0, y_{1}(0)=0, \dot{y}_{1}=0$; $\ddot{y}_{2}+\dot{y}_{1}=0, y_{2}(0)=0, \dot{y}_{2}(0)=0$, on equating the coefficients of $\epsilon^{i}$. Solving these equations, we get $y_{0}(x)=x-\frac{x^{2}}{2}, y_{1}(x)=-\frac{x^{2}}{2}+\frac{x^{3}}{6}, y_{2}(x)=\frac{x^{3}}{6}-\frac{x^{4}}{24}$, in conformation with the results obtained using Taylor's series expansion [8].

\section{Example 6}

Consider a right angle triangle with sides $1,0.1$, and $1.0049 \cong \sqrt{1^{2}+0.1^{2}}$, it is difficult to differentiate between this right angle triangle and its being an isosceles one; since there is not that difference between the two sides 1 and 1.0049 . Let us make a simulation of the situation using perturbation theory by considering the side as $1, \epsilon$ and $\sqrt{1+\epsilon^{2}}$. Using binomial theorem we have $\sqrt{1+\epsilon^{2}}=1+\epsilon^{2}+O\left(\epsilon^{4}\right)$. Hence, the difference between the side 1 and the hypotenuse is of order $\epsilon^{2}(=0.01)$ which is a small number. Note that the angle between them is given by $\theta=\tan ^{-1}\left(\frac{\epsilon}{1}\right) \cong \epsilon=5.71^{\circ}$ which is a sensible angle and can be observed better than the difference between the side and the hypotenuse. Actually, if we make the value of $\epsilon$ smaller, we will be misled to the conclusion that the triangle is segment of a straight line [9].

\subsection{The Smallness of the Perturbation Parameter and Its Relation with the Unperturbed Operator}

If we consider the following eigenvalue problem

$$
-\frac{\mathrm{d}^{2} u}{\mathrm{~d} x^{2}}+a^{2} x^{2} u=\lambda u,-\infty<x<\infty
$$

where $a$ is a given positive constant, then the discrete eigenvalues are given by

$$
\lambda_{n}=(2 n+1) a, n=0,1,2,3, \cdots
$$

And the corresponding eigenfunctions are

$$
u_{n}=\mathrm{e}^{-a x^{2} / 2} H_{n}(\sqrt{a} x), n=0,1,2,3, \cdots
$$


$H_{n}(x)=(-1)^{n} \mathrm{e}^{x^{2}} \frac{\mathrm{d}^{2}}{\mathrm{~d} x^{2}}\left(\mathrm{e}^{x^{2}}\right)$ is a Hermite polynomial.

This problem describes the eigenvalue problem for the simple harmonic oscillator [10].

Now, adding a perturbation term of the form $\epsilon x u$, we get

$$
-\frac{\mathrm{d}^{2} u}{\mathrm{~d} x^{2}}+a^{2} x^{2} u+\epsilon x u=\lambda u,-\infty<x<\infty
$$

We may look at the term $\epsilon x u$ as not small. This is because the variable value $x$ takes values from $-\infty$ to $\infty$; however if we compare this perturbation parameter with the term $a^{2} x^{2} u$ which is the non-perturbed one, the perturbation parameter (for small $\epsilon$ ) is then considered as a small perturbation. One concludes then that: In perturbation problems, the perturbing parameter has to be small in comparison with the unperturbed operator [6].

Now the eigenvalues and eigenfunctions for the perturbed problem are given by

$$
\lambda_{n}=(2 n+1) a-\frac{\epsilon^{2}}{4 a^{2}}, n=0,1,2,3, \cdots
$$

and

$$
u_{n}=\mathrm{e}^{-a / 2\left(x+\frac{\epsilon}{2 a^{2}}\right)^{2}} H_{n}\left(\sqrt{a}\left[x+\frac{\epsilon}{2 a^{2}}\right]\right), n=0,1,2,3, \cdots
$$

Note that the functions $u_{n}$ are regular functions in $\epsilon$.

At the end of this subsection, we note that the main goal of perturbation theory is the precise definition of smallness of the perturbing parameter with respect to the unperturbed operator [6].

Before we move to the applications of perturbation theory in different applied fields, we make a little digression, in the next section, on spectral perturbation theory.

\subsection{Spectral Perturbation Theory}

The theory deals with the way the perturbation affects the spectrum of a certain operator when it is perturbed. This compels us to reformulate the problem in a more precise and different manner [6].

\section{Definition 1}

If $\mathbb{H}$ is a Hilbert space with inner product $\langle.,$.$\rangle and that A^{\epsilon}$ is a linear operator in $\mathbb{H}$ such that $D\left(A^{\epsilon}\right) \subset \mathbb{H} \rightarrow \mathbb{H}$ and $D\left(A^{\epsilon}\right)$ is the domain of $A^{\epsilon}$ which depends on a small parameter $\epsilon$; assume that

1) $A^{\epsilon}$ Is a self-adjoint operator, i.e.

$$
\left\langle x, A^{\epsilon} y\right\rangle=\left\langle A^{\epsilon} x, y\right\rangle, \forall x, y \in D\left(A^{\epsilon}\right)
$$

2) $A^{\epsilon}$ has a smooth branch of simple eigenvalues $\lambda^{\epsilon} \in R$ with eigenvectors $x^{\epsilon} \in \mathbb{H}$; namely

$$
A^{\epsilon} x^{\epsilon}=\lambda^{\epsilon} x^{\epsilon}
$$


Then, the job of perturbation theory is the calculation of the eigenvalues with respect to their values at $\epsilon=0$ when $\epsilon$ is small but not equal to zero [6].

To clarify this idea, we take as an example the perturbation in the eigenvalues for a symmetric matrix; in this case $\mathbb{H}=R^{n}$, with the Euclidean inner product

$$
\langle x, y\rangle=x^{\mathrm{T}} y
$$

And $A^{\epsilon}: R^{n} \rightarrow R^{n}$ is a linear transformation for a symmetric matrix of the order $n \times n\left(a_{i j}^{\epsilon}\right)$. [note that we are concentrating on symmetric matrices because these are what we face in physics]

Moreover, the perturbation in the eigenvalues for a Hermitian matrix is of our concern, in this case $\mathbb{H}=C^{n}$ with the inner product

$$
\langle x, y\rangle=\bar{x}^{\mathrm{T}} y
$$

Problems of this sort are found in many applications especially in quantum mechanics and where Hilbert spaces of infinite dimensions are dealt with. In this case expansions of the form

$$
\begin{aligned}
& A^{\epsilon}=A_{0}+\epsilon A_{1}+\cdots+\epsilon^{n} A_{n}+\cdots, \\
& x^{\epsilon}=x+\epsilon x_{1}+\cdots+\epsilon^{n} x_{n}+\cdots, \\
& \lambda^{\epsilon}=\lambda_{0}+\epsilon \lambda_{1}+\cdots+\epsilon^{n} \lambda_{n}+\cdots
\end{aligned}
$$

Are used.

Now following the same techniques we have used in solving previous examples and in particular equating coefficients of $\epsilon^{n}$, we get the following equations

$$
\begin{gathered}
\left(A_{0}-\lambda_{0} I\right) x_{0}=0 \\
\left(A_{0}-\lambda_{0} I\right) x_{1}=-A_{1} x_{0}+\lambda_{1} x_{0} \\
\cdots \\
\left(A_{0}-\lambda_{0} I\right) x_{n}=\sum_{i=1}^{n}\left(-A_{i} x_{n-i}+\lambda_{i} x_{n-i}\right)
\end{gathered}
$$

[ $I$ is the $n \times n$ unit matrix].

Now, suppose that $x_{0} \neq 0$, then from Equation (35) $\lambda_{0}$ is the eigenvalue of the unperturbed operator $A_{0}$; and the corresponding eigenvector is $x_{0}$ [7].

As to the second correction $\lambda_{1}$ and $x_{1}$, we note that Equation (36) is a singular equation and in order to be able to solve it, we need a certain hypothesis and the so-called Fredholm alternative [7].

\section{Applications}

In this section, we give some representative well-known problems in different branches of physics; we start first with the ones from classical physics, then we move to the ones from modern physics (or more specific in quantum mechanics).

\subsection{Problems from Classical Physics}

\section{1) String Problem in the Theory of Sound}


The vibrations of a string, with a small stiffness can be described the following eigenvalue problem

$$
-\frac{\mathrm{d}^{2} u}{\mathrm{~d} x^{2}}+\epsilon \frac{\mathrm{d}^{4} u}{\mathrm{~d} x^{4}}=\lambda u, 0<x<1
$$

where $\epsilon$ is a small positive number and is proportional to the stiffness, i.e. $\epsilon$ represents the perturbation. To solve this problem boundary conditions are needed. For instance to impose the conditions at the end points; $x=1$ and $x=0 \quad$ [6].

If the two ends of the string are to be supported, then the boundary conditions will be $u(0)=\ddot{u}(0)=0, u(1)=\ddot{u}(1)=0$; with these conditions in mind, one can get the eigenfunctions and eigenvalues as

$$
u_{n}(x)=\sin n \pi x \text { and } \lambda_{n}=n^{2} \pi^{2}+\epsilon n^{4} \pi^{4}, n=1,2,3, \cdots
$$

The set $\{\sin n \pi x\}_{n=1}^{\infty}$ is complete in the interval $(0,1)$; this means that $\left\{\lambda_{n}\right\}_{n=1}^{\infty}$ represents the full spectrum of the problem. For $\epsilon \geq 0$, the spectrum is bounded below and the smallest value of the eigenvalues is $\lambda_{1}=\pi^{2}+\epsilon \pi^{4}$. However, for $\epsilon<0$ the spectrum is not bounded below and this is not allowed because $\epsilon$ measure the stiffness which is a physical quantity and is not negative.

\section{2) The Simple Pendulum}

The equation of motion for the simple pendulum is

$$
m L \ddot{\theta}+m g \sin \theta=0
$$

where $\theta$ is the angle between the light string (of length $L$ ) and the vertical equilibrium position, $m$ is the mass, and $g$ is the gravitational acceleration. The equation can be written as

$$
\ddot{\theta}+k^{2} \sin \theta=0
$$

where $k^{2}=g / L$.

Now, taking into account the initial conditions, the problem, for a small angle, then becomes

$$
\ddot{\theta}+k^{2} \sin \theta=0, \theta(0)=\epsilon, \dot{\theta}(0)=0
$$

And $0<\epsilon \ll 1, \theta=O(\epsilon)$.

Putting $\theta=\epsilon \Psi$ and dividing by $\epsilon$, the last equation can be written as

$$
\ddot{\Psi}+k^{2}\left(\Psi-\frac{1}{6} \epsilon^{2} \Psi^{3}\right)=0, \Psi(0)=1, \dot{\Psi}=0
$$

If the solution is required to be of second order, then the higher order terms can be neglected. Making the transformation $\tau=\omega t$, where $t$ is the time, Equation (43) is then written as

$$
\omega^{2} \ddot{\Psi}+k^{2}\left(\Psi-\frac{1}{6} \epsilon^{2} \Psi^{3}\right)=0
$$

And where the differentiation is with respect to $\tau$. Now, expanding in terms of powers of $\epsilon^{2}$, we get 


$$
\omega=\omega_{0}+\epsilon^{2} \omega_{1}
$$

And

$$
\Psi=\Psi_{0}+\epsilon^{2} \Psi_{1}
$$

Substituting with Equations (45)-(46) in Equation (44) and equating coefficients of $\epsilon^{2}$, one obtains

$$
\omega_{0} \ddot{\Psi}_{0}+k^{2} \Psi_{0}=0, \Psi_{0}=1, \dot{\Psi}_{0}=0
$$

And

$$
\omega_{0} \ddot{\Psi}_{1}+k^{2} \Psi_{1}=-2 \omega_{0} \omega_{1} \ddot{\Psi}_{0}+\frac{1}{6} k^{2} \Psi_{0}^{3} ; \quad \Psi_{1}(0)=0, \quad \dot{\Psi}_{1}(0)=0
$$

Choosing $\omega_{0}=k$, the solution $\Psi_{0}$ is given by

$$
\Psi_{0}=\cos \tau ; \omega_{0}=k,
$$

while $\Psi_{1}$ satisfies the equation

$$
\ddot{\Psi}_{1}+\Psi_{1}=\frac{2}{k} \omega_{1} \cos \tau+\frac{1}{8} \cos \tau+\frac{1}{24} \cos 3 \tau
$$

If we choose $\omega_{1}=-\frac{k}{16}$ the solution $\Psi_{1}$ will then be

$$
\Psi_{1}=\frac{1}{192}(\cos \tau-\cos 3 \tau)
$$

and the final solution is

$$
\theta(t)=\epsilon \cos \tau+\frac{1}{192} \epsilon^{3}(\cos \tau-\cos 3 \tau)+O\left(\epsilon^{5}\right)
$$

Moreover, $\tau=k\left[1-\frac{1}{16} \epsilon^{2}+O\left(\epsilon^{4}\right)\right][11]$.

\section{3) Steady State Diffusion Through a Corrugated Slab}

In this application, the simplest type of heat transfer is dealt with, which is the steady state type, i.e. when $\frac{\partial T}{\partial t}=0$. The flow of heat is through a corrugated slab of thickness $H$, with the assumption that the lower side $(z=0)$ is at temperature $T_{0}$; and the upper side $(z=H)$ is at temperature $T_{0}+\Delta T$. Then, for steady state flow we have

$$
k T_{z z}=0
$$

(this is because the temperature does not depend on time explicitly $\frac{\partial T}{\partial t}=0$ ).

From the boundary conditions, the solution is given by

$$
T=T_{0}+\frac{\Delta T}{H} z
$$

And the heat flux through the slab is determined by

$$
\vec{F}=-\frac{k \Delta T}{H} \frac{\vec{z}}{z}
$$

Now, assuming that the slab occupies the region $-\infty<x<\infty, h(x)<z<H$, 
and suppose that the slab is not homogenous with a boundary condition on the lower part at $z=h(x)$ given by

$$
h(x)=h_{\max } \cos k x
$$

where $h_{\max }<H$; in this case Laplace equation for the steady state case is given by

$$
T_{x x}+T_{z z}=0
$$

with Dirichlet boundary conditions given as

$$
T(x, h(x))=T_{0} ; T(x, H)=T_{0}+\Delta T
$$

Introducing the dimensionless variables

$$
\bar{T}=\frac{T-T_{0}}{\Delta T}, \bar{x}=k x, \bar{z}=k z
$$

The problem becomes dimensionless and the boundary conditions will be as

$$
T(x, \epsilon \cos x)=0, T(x, \beta)=1
$$

where $\epsilon=k h_{\max }, \beta=k H$. It is clear that $h_{\max }<H$ and hence $\epsilon<\beta$.

For small amplitudes, $\epsilon \ll 1$, and this is fulfilled when $h_{\max }$ is made to be very small ( $\epsilon \rightarrow 0$ with $\beta$ kept as a constant) [12].

Now, the boundary condition $T(x, \epsilon h)=0$ can be written as a Taylor series as

$$
T(x, 0)+\epsilon h T_{z}(x, 0)+\frac{1}{2}(\epsilon h)^{2} T_{z z}(x, 0)+\cdots
$$

Also, using perturbation theory, $T$ is written as

$$
T=T_{0}+\epsilon T_{1}+\epsilon^{2} T_{2}+\cdots
$$

Using the last two equations, following the same old procedure, and equating coefficients of $\epsilon^{n}$, we get

$$
T_{0}=0, T_{1}+h T_{0 z}=0, T_{2}+h T_{1 z}+\frac{1}{2} h^{2} T_{0 z z}=0
$$

This is for the first boundary condition; as for the second one $T(x, \beta)=1$, it tends to $T_{0}=1$ and $T_{n \geq 1}=0$, with all $T_{n}(x, z)$ satisfying Laplace equation $\left(\frac{\partial^{2}}{\partial x^{2}}+\frac{\partial^{2}}{\partial z^{2}}\right) T_{n}=0$. putting, now $h=\cos x$; we get the solution as

$$
\begin{aligned}
T= & \frac{z}{\beta}-\epsilon \cos x \frac{\sinh (\beta-z)}{\beta \sinh h \beta} \\
& -\frac{1}{2} \epsilon^{2} \frac{\operatorname{coth} \beta}{\beta}\left[\frac{\beta-z}{\beta}+\cos 2 x\left(\frac{\sinh 2(\beta-z)}{\sinh 2 \beta}\right)\right]+O\left(\epsilon^{3}\right)
\end{aligned}
$$

\section{4) Incompressible Flow Past a Circle}

Here, the stream function is given by

$$
\mathrm{d} \Psi=u \mathrm{~d} y-v \mathrm{~d} x
$$

$(u, v)$ is the velocity; and if the motion is inviscid, the function satisfies the eq- 
uation

$$
\nabla^{2} \Psi=-\omega(\Psi)
$$

where $\omega$ is the vorticity, which is equal to zero upstream if the flow is uniform; hence Equation (66) becomes, in cylindrical coordinates, as

$$
\Psi_{r r}+\frac{1}{r} \Psi_{r}+\frac{1}{r^{2}} \Psi_{\theta \theta}=0
$$

Also, the boundary conditions are given by

$$
\Psi(r, \theta) \rightarrow u r \sin \theta \text { as } r \rightarrow \infty \quad \text { (upstream) }
$$

and

$$
\Psi(a, \theta)=0 \quad \text { (on the surface) }
$$

where $a$ is the radius of the circle. Moreover, it is assumed that there is no rotational motion, this means that the flow should be symmetrical about the line $\theta=0$ and this means that $\Psi(r, \theta)=-\Psi(r,-\theta)$; hence the solution, in this case, will be

$$
\Psi_{0}=u\left(r-\frac{a^{2}}{r}\right) \sin \theta
$$

Now, in the presence of the circle and if the vorticity number $\epsilon$ is small, then we expect that the solution will not depart from the one we got in Equation (70). So, we write the solution in the presence of vorticity as

$$
\Psi(r, \theta ; \epsilon)=\Psi_{0}(r, \theta)+\epsilon \Psi_{1}(r, \theta)
$$

$\Psi_{0}(r, \theta)$ is the unperturbed solution given by Equation (70).

On substituting Equation (71) in Equation (67), and equating coefficients of $\epsilon^{n}$, we get

$$
\frac{\partial^{2} \Psi_{1}}{\partial r^{2}}+\frac{1}{r} \frac{\partial \Psi_{1}}{\partial r}+\frac{1}{r^{2}} \frac{\partial^{2} \Psi_{1}}{\partial \theta^{2}}=\frac{u}{a}
$$

Also, we get the boundary conditions as

$$
\Psi_{1} \rightarrow \frac{1}{4} \frac{u}{a} r^{2}(1-\cos 2 \theta) ; r \rightarrow \infty, \Psi_{1}(a, \theta)=0
$$

Therefore, the solution is written as

$$
\Psi_{1}=\frac{1}{4} \frac{u}{a} r^{2}(1-\cos 2 \theta)+\varphi(r, \theta)
$$

And the equation in $\varphi(r, \theta)$ is given by

$$
\frac{\partial^{2} \varphi_{1}}{\partial r^{2}}+\frac{1}{r} \frac{\partial \varphi_{1}}{\partial r}+\frac{1}{r^{2}} \frac{\partial^{2} \varphi_{1}}{\partial \theta^{2}}=0
$$

With the conditions

$$
\varphi_{1} \rightarrow \text { constant as } r \rightarrow \infty ; \varphi_{1}(a, \theta)=-\frac{1}{4} u a(1-\cos 2 \theta)
$$

Using separation of variables, the solution in final form is 


$$
\Psi=u\left(r-\frac{a^{2}}{r}\right) \sin \theta+\frac{1}{4} \epsilon u u\left[\frac{r^{2}}{a}(1-\cos 2 \theta)+\frac{a^{3}}{r^{2}} \cos 2 \theta-a\right]
$$

Equation (77) gives the solution in first order in $\epsilon$ [12].

\subsection{Problems in Modern Physics}

In some physics subjects, one can solve for the eigenvalues and eigenfunctions of the Hamiltonian of the problem exactly and precisely such as the simple harmonic oscillator and the Hydrogen atom in atomic physics. However, many physical systems in nature cannot be solved exactly and that requires the need for the development of appropriate procedures and tools to deal with such situations. As we stated before, perturbation theory was successful in treating these cases in which the distortion in the system, that can be solved exactly, is small.

Mathematically, the Hamiltonian of the system $\boldsymbol{H}$ is written as

$$
\boldsymbol{H}=\boldsymbol{H}_{0}+\epsilon \boldsymbol{V}
$$

$\boldsymbol{H}_{0}$ is the unperturbed part of the Hamiltonian, $\epsilon$ is a small parameter, and $\boldsymbol{V}$ is the potential, related to the perturbation, and supposed to be independent of time.

We assume that we can solve the time-independent Schrodinger equation for the unperturbed Hamiltonian i.e. we can get the eigenvalues $E_{n}$ and the eigenfunctions $\Psi_{n}(x)$, such that

$$
\boldsymbol{H}_{0} \Psi_{n}(x)=E_{n} \Psi_{n}(x)
$$

Such solutions may give unperturbed eigenvalues $E_{n}$, nondegenerate or degenerate.

\subsection{Nondegenerate Solutions}

Let the time-independent Schrodinger equation for the total Hamiltonian be

$$
\boldsymbol{H} \Psi(x)=E \Psi(x)
$$

Since $\epsilon$ is a small parameter, we can use Taylor series to expand the wave function $\Psi(x)$ in terms of $\epsilon$ as

$$
\Psi(x)=\Psi_{0}(x)+\epsilon \eta_{1}(x)+\epsilon^{2} \eta_{2}(x)+\cdots
$$

The same thing can be done with the state energy to get

$$
E=E_{0}+\epsilon \mathcal{E}_{1}+\epsilon^{2} \mathcal{E}_{2}+\cdots
$$

Substituting with these two equations in Equation (81) one gets

$$
\begin{aligned}
& \left(\boldsymbol{H}_{0}+\epsilon \boldsymbol{V}\right)\left(\Psi_{0}(x)+\epsilon \eta_{1}(x)+\epsilon^{2} \eta_{2}(x)+\cdots\right) \\
& =\left(E_{0}+\epsilon \mathcal{E}_{1}+\epsilon^{2} \mathcal{E}_{2}+\cdots\right)\left(\Psi_{0}(x)+\epsilon \eta_{1}(x)+\epsilon^{2} \eta_{2}(x)+\cdots\right)
\end{aligned}
$$

$\left[\left(\mathcal{E}_{1}, \eta_{1}\right),\left(\mathcal{E}_{2}, \eta_{2}\right), \cdots\right.$ are the corrections in $E$ and $\Psi$ ].

Comparing terms of $\epsilon^{n}$, one gets

For the order $\epsilon^{0}$

$$
\left(\boldsymbol{H}_{0}-E_{0}\right) \Psi_{0}=0
$$


This equation actually shows that $\Psi_{0}$ is the unperturbed eigenfunction corresponding to the eigenvalue $E_{0}$.

For the order $\epsilon^{1}$

$$
\left(\boldsymbol{H}_{0}-E_{0}\right) \eta_{1}+\left(\boldsymbol{V}-\mathcal{E}_{1}\right) \Psi_{0}=0
$$

Taking the scalar product of this equation with $\Psi_{0}$, one gets

$$
\left\langle\Psi_{0}, \boldsymbol{H}_{0}, \eta_{1}\right\rangle+\left\langle\Psi_{0}, V \Psi_{0}\right\rangle=E_{0}\left\langle\Psi_{0}, \eta_{1}\right\rangle+\mathcal{E}_{1}\left\langle\Psi_{0}, \Psi_{0}\right\rangle
$$

Using the hermiticity of $\boldsymbol{H}_{0}$ and that $E_{0}$ is the eigenvalue of $\boldsymbol{H}_{0}$, one gets

$$
\mathcal{E}_{1}=\frac{\left\langle\Psi_{0}, V \Psi_{0}\right\rangle}{\left\langle\Psi_{0}, \Psi_{0}\right\rangle}
$$

$\mathcal{E}_{1}$ represents the shift in $E$ to first order; which means that

$$
E=E_{0}+\epsilon \frac{\left\langle\Psi_{0}, V \Psi_{0}\right\rangle}{\left\langle\Psi_{0}, \Psi_{0}\right\rangle}+O\left(\epsilon^{2}\right)
$$

This is a very important result which can be stated as

\{The perturbed energy levels can be calculated from those which are not perturbed [13].

Now, taking the scalar product of Equation (86) with $\Psi_{m}$ and using the orthogonality of the eigenfunctions, and the hermiticty of the unperturbed Hamiltonian, one obtains

$$
\left\langle\Psi_{m}, \eta_{1}\right\rangle=\frac{\left\langle\Psi_{m}, V \Psi_{0}\right\rangle}{E_{0}-E_{m}}
$$

Hence and in first order, the perturbed eigenfunction is given by

$$
\Psi(x)=\Psi_{0}(x)+\epsilon \sum_{m \neq 0} \frac{\left\langle\Psi_{m}, V \Psi_{0}\right\rangle}{E_{0}-E_{m}} \Psi_{m}(x)+O\left(\epsilon^{2}\right)
$$

This result is for the ground state; a similar result can be obtained for the other energy levels $E_{n}$ and

$$
\Psi(x)=\Psi_{n}(x)+\epsilon \sum_{m \neq n} \frac{\left\langle\Psi_{m}, V \Psi_{n}\right\rangle}{E_{n}-E_{m}} \Psi_{m}(x)+O\left(\epsilon^{2}\right)
$$

Note that $\Psi(x)$ is normalized to first order in $\epsilon$, namely $\langle\Psi \mid \Psi\rangle=1+O\left(\epsilon^{2}\right)$; where Dirac notation is used here.

\section{Example 7. Infinite Square Well}

Consider a particle moving under the potential described as

$$
V(x)=\left\{\begin{array}{ll}
V_{0} \cos \left(\frac{\pi x}{2 a}\right), & |x|<a \\
\infty, & |x| \geq a
\end{array}\right. \text {, and assume that it is required to compute the }
$$

ground state energy to first order using perturbation theory. Then, to do that we need to seek solutions of the corresponding infinite square well problem. The potential in this case is given by $V_{p}(x)=\left\{\begin{array}{l}0,|x|<a \\ \infty,|x| \geq a\end{array}\right.$; this means that the particle $(m)$ is moving inside a box $(|x|<a)$ freely, while there is a very strong repulsion 
force at the walls $(x= \pm a)$. Hence, the time-independent Schrodinger equation is given by $E u=-\frac{\hbar^{2}}{2 m} \frac{\mathrm{d}^{2} u}{\mathrm{~d} x^{2}}$, its solution is then $u_{n}(x)=\frac{1}{\sqrt{a}}\left\{\begin{array}{l}\cos \left(\frac{n \pi x}{2 a}\right), \text { odd } n \\ \sin \left(\frac{n \pi x}{2 a}\right), \text { even } n\end{array}\right.$; moreover, the energy eigenvalues are given by $E_{n}=\frac{n^{2} \pi^{2} \hbar^{2}}{8 m a^{2}}$; and the least value energy ( ground state) is $E_{1}=\frac{\pi^{2} \hbar^{2}}{8 m a^{2}}$ [13].

Now, going back to the original problem, we see that the ground state energy needs to be calculated to first order where the perturbation is given by $H^{\prime}=V_{0} \cos \left(\frac{\pi x}{2 a}\right)$; this is small compared with the term $E_{2}-E_{1}$.

Or $V_{0} \ll E_{2}-E_{1}$. Using perturbation theory techniques, one obtains the shift in ground state energy as

$$
\Delta E=\mathcal{E}_{1}=H_{11}^{\prime}=\int_{-\infty}^{\infty} u_{1}^{*} H^{\prime} u_{1} \mathrm{~d} x=\frac{V_{0}}{a} \int_{-a}^{a} \cos ^{3}\left(\frac{\pi x}{2 a}\right) \mathrm{d} x=\frac{8 V_{0}}{3 \pi}=0.85 V_{0}
$$

(it is to be noted that $n=1$ for the ground state energy) [13] [14].

\section{Example 8. Simple Harmonic Oscillator under a Linear Perturbation}

The unperturbed Hamiltonian for the simple harmonic oscillator in one dimension is given by

$$
\boldsymbol{H}_{0}=-\frac{\hbar^{2}}{2 m} \frac{\mathrm{d}^{2}}{\mathrm{~d} x^{2}}+\left(\frac{m \omega_{0}^{2}}{2}\right) x^{2}=\frac{1}{2}\left(-\frac{\mathrm{d}^{2}}{\mathrm{~d} \rho^{2}}+\rho^{2}\right) \hbar \omega_{0},
$$

where $\rho=\sqrt{\frac{m \omega_{0}}{\hbar}} x$. Putting $\hbar \omega_{0}=1$, one gets for the unperturbed states energy $E_{n}^{(0)}=n+\frac{1}{2}, n=0,1,2, \cdots$; the superscript 0 means that the energy is for the unperturbed case. Also, the unperturbed normalized eigenfunctions are given by $\Psi_{n}^{(0)}(\rho)=N_{n} H_{n}(\rho) \mathrm{e}^{-\frac{\rho^{2}}{2}} ; N_{n}=\pi^{-1 / 4}\left(2^{n} n !\right)^{2} ; H_{n}(\rho)$ are Hermite polynomials.

Now, in the presence of the linear perturbation $\beta \rho$, we need to compute the matrix elements of $\rho$; which is $\langle n|\rho| m\rangle=\int_{-\infty}^{\infty} \Psi_{n}^{(0)}(\rho) \rho \Psi_{m}^{(0)}(\rho) \mathrm{d} \rho$; again Dirac notation is used here for the eigenfunctions. Using the recurrence relation for Hermite polynomials [10], $H_{n+1}(\rho)=2 \rho H_{n}(\rho)-2 n H_{n-1}(\rho)$, and the form of $\Psi_{n}^{(0)}(\rho)$, one obtains that $\rho \Psi_{n}^{(0)}(\rho)=\sqrt{\frac{n+1}{2}} \Psi_{n+1}^{(0)}(\rho)+\sqrt{\frac{n}{2}} \Psi_{n-1}^{(0)}(\rho)$. Taking the product of this equation with $\Psi_{m}^{(0)}(\rho)$ and using the orthogonality of these functions, we get $\langle m|\rho| n\rangle=\langle n|\rho| m\rangle=\delta_{m, n+1} \sqrt{\frac{n+1}{2}}+\delta_{m, n-1} \sqrt{\frac{n}{2}}$; and hence, for a linear perturbation of the form $H^{\prime}=\beta \rho$, the total Hamiltonian is $H=H_{0}+H^{\prime}=\frac{1}{2}\left(-\frac{\mathrm{d}^{2}}{\mathrm{~d} \rho^{2}}+\rho^{2}\right)+\beta \rho=\frac{1}{2}\left(-\frac{\mathrm{d}^{2}}{\mathrm{~d} \mu^{2}}+\mu^{2}\right)-\frac{1}{2} \beta^{2}$; where $\mu=\rho+\beta$. 
Therefore, the total energy, including the perturbation term, is given by $E_{n}=E_{n}^{(0)}-\frac{1}{2} \beta^{2}=n+\frac{1}{2}-\frac{1}{2} \beta^{2} \quad[14]$.

\subsection{Degenerate States}

The correction in the eigenfunctions, in first order of perturbation, is small only when

$$
\frac{\left\langle\Psi_{m}|V| \Psi_{n}\right\rangle}{E_{n}-E_{m}} \cong 1
$$

If the difference between the unperturbed energy eigenvalues is small compared with the matrix element in the numerator $V_{m n}$, then the perturbation will be large and the approximation fails. In particular, if there exist levels of the degenerate type (levels with the same energy), the denominator will be equal to zero which is a singular situation and the solution obtained for the nondegenerate states is not acceptable.

Now, if unperturbed state is $l$-fold degenerate, then we use the projection $\boldsymbol{P}$ on the state and the projection $\boldsymbol{Q}$ perpendicular to it. Acting with $\boldsymbol{P}$ on the space of these degenerate states, one gets

$$
\boldsymbol{P}\left(\boldsymbol{V}-\mathcal{E}_{1}\right) \Psi_{0}=0
$$

Choosing an appropriate basis for the space of the degenerate states $\left\{\varnothing_{i}\right\}_{i=1}^{l}$; $\Psi_{0}$ can be written as

$$
\Psi_{0}=\sum_{i=1}^{l} c_{i} \varnothing_{i}
$$

So, one gets

$$
\left\langle\varnothing_{i}|V| \varnothing_{j}\right\rangle c_{j}=\mathcal{E}_{1} c_{i}
$$

Hence, $\mathcal{E}_{1}$ is an eigenvalue of the matrix $\left(V_{i j}\right)$. this will lead to 1 roots. Equation (96) is a generalization of Equation (89) for the nondegenerate case. Moreover, if the states are degenerate, one can, partially, remove the degeneracy as will be shown in the following example.

\section{Example 9. Stark Effect}

The Schrodinger equation for the Hydrogen atom is given by [10]

$$
-\frac{\hbar^{2}}{2 m} \nabla^{2} u-\frac{e^{2}}{r} u=E u
$$

$m, e$ are the mass and charge of the electron which is orbiting around the proton.

Equation (96) can be written as $\boldsymbol{H} u=E u$, where $\boldsymbol{H}$ is the Hamiltonian and $u$ is the eigenfunction which describes the electron motion under the attractive force $-\frac{e^{2}}{r}$; while $E$ is the eigenvalue (the energy). Using separation of variables in spherical coordinates, the eigenvalues are found to be [10]

$$
E_{n}=-\frac{m e^{4}}{2 n^{2} \hbar^{2}}, n=1,2,3, \cdots
$$


$n$ is the principal quantum number and for each $n$ there exist an orbital angular quantum number $l$ such that $l<n$ and $l=0,1,2, \cdots, n-1$; also, there exist a magnetic quantum number $m$ such that $-l \leq m \leq l$. This means that for each orbital quantum number $l$ the degeneracy in energy states equals $2 l+1$.

The state with $l=0$ is labelled as $s$-state, while the one with $l=1$ is called the $p$-state, ... and so on. Moreover, the ground eigenstate for Hydrogen atom is given $u_{100}(\vec{r})=\frac{1}{\sqrt{\pi}}\left(\frac{1}{a_{0}}\right)^{\frac{3}{2}} \exp \left(-\frac{r}{a}\right)$ [10]. Note that, in this case, $n=1 \rightarrow l=0=m$. In terms of the fine structure formula, the wave function in this situation is expressed as $|1 s\rangle$. If $n=2$, then $l=0,1$ and there is a 4 -fold degeneracy with the same energy and the eigenstates are $|2 s\rangle,|2 p, 0\rangle,|2 p,+1\rangle$, $|2 p,-1\rangle$,

Now, if the Hydrogen atom is acted upon by a weak electric field $\vec{E}$ in the z-direction, and $\vec{E}=\mathbb{E} \hat{z} ; \mathbb{E}$ is the magnitude of the electric field. The potential, in this case, is $V=-e z \mathbb{E}$.

To compute the matrix elements of $V_{i j}$, for the unperturbed states for the Hydrogen atom with energy $E_{2}$, we note that we are computing a $4 \times 4$ Hermitian matrix keeping in mind that the perturbing potential is odd under parity operation; and hence the only non-zero elements are those with different parity (e.g. $V_{01} \neq 0$, and $V_{10} \neq 0$, this because $|2 s\rangle$ is of even parity, while $|2 p, 0\rangle$ is of odd parity). Note, also that the Hydrogen eigenstates are eigenstates of the total angular momentum $\boldsymbol{L}^{2}$ and the $z$-component of the angular momentum $\boldsymbol{L}_{z}$. Therefore, $V_{i j}$ between states $p$ and $s$ may not be zeros; moreover $V$ commutes with $\boldsymbol{L}_{z}$. Taking previous information into account, the only non-zero elements are $\langle 2 s|\boldsymbol{V}| 2 p, 0\rangle$ and its Hermitian conjugate. i.e.

$$
\left(V_{i j}\right)=\left(\begin{array}{cccc}
0 & 3 e \mathbb{E} a_{0} & 0 & 0 \\
3 e \mathbb{E} a_{0} & 0 & 0 & 0 \\
0 & 0 & 0 & 0 \\
0 & 0 & 0 & 0
\end{array}\right) \text {. Note that to reach these results, the forms of }
$$

the Hydrogen eigenstates were used; $a_{0}$ is Bohr radius. From these results we conclude that the electric field was able to remove the degeneracy between the two states $|2 p, 0\rangle$ and $|2 s\rangle$ (it made what was hidden apparent); while the other two states $|2 p, \pm 1\rangle$ are still without any change in their status. What happened for the states $|2 s\rangle$ and $|2 p, 0\rangle$ was a discretization in the energy, so that the energy of one of them becomes $E_{0}+3 e \mathbb{E} a_{0}$ and the other is $E_{0}-3 e \mathbb{E} a_{0}$. This is the so-called Stark effect [13].

Example 10. The Approximation in Calculating the Ground State Energy for the Helium Atom

In the He atom, the nucleus is composed of two protons and two neutrons with two electrons moving around the nucleus. The unperturbed total Hamiltonian is given by $\boldsymbol{H}_{0}=\boldsymbol{H}_{1}+\boldsymbol{H}_{2}$ where $\boldsymbol{H}_{i}, i=1,2$ are the Hamiltonians for the first and second electrons; the total, ground state, eigenfunction of the total unperturbed Hamiltonian is given by $\Psi_{0}=u_{100}\left(\vec{r}_{1}\right) u_{100}\left(\vec{r}_{2}\right) \psi_{0,0} ; \vec{r}_{1}, \vec{r}_{2}$ are the po- 
sition vectors for electron 1 and electron 2 respectively. While, $\psi_{0,0}$ has to do with the spin of the ground state.

For the total Hamiltonian $\boldsymbol{H}$, when the repulsion between the two electrons is taken into account $\left(\boldsymbol{H}^{\prime}\right)$ and which is considered as the perturbation in the problem, is given by $\boldsymbol{H}=\boldsymbol{H}_{0}+\boldsymbol{H}^{\prime}$ and $\boldsymbol{H}^{\prime}=\frac{e^{2}}{4 \pi \epsilon_{0} r_{12}}, \quad r_{12}=\left|\vec{r}_{1}-\vec{r}_{2}\right|$.

Now, to compute the first order correction in the energy, we compute the expectation value for $\boldsymbol{H}^{\prime}$, and where $u_{100}(\vec{r})=\frac{1}{\sqrt{\pi}}\left(\frac{Z}{a_{0}}\right)^{\frac{3}{2}} \exp \left(-\frac{Z r}{a_{0}}\right)$; hence $\Delta E_{1}=\frac{a_{0}}{4 \pi \epsilon_{0}}\left(\frac{Z^{3}}{\pi a_{0}^{3}}\right)^{2} \int \frac{1}{r_{12}} \exp \left(-\frac{2 Z\left(r_{1}+r_{2}\right)}{a_{0}}\right) \mathrm{d} \tau_{1} \mathrm{~d} \tau_{2}=\frac{5}{4} Z R_{y}=34 \mathrm{eV} ; \quad(Z=2 \quad$ is the atomic number for Helium and $R_{y}[=13.606 \mathrm{eV}]$ is Rydberg constant) [13].

Therefore, $E_{1}=-108.8 \mathrm{eV}+34 \mathrm{eV}=-74.8 \mathrm{eV}$, and this represents the corrected value of the energy, in first order, for the Helium ground state [13] [15].

Example 11. Zeeman Effect

In this problem, an electron $(-e)$ moves under the effect of a field resulting from a nucleus with positive charge $Z e$ positioned at the center of the coordinates system $x, y, z$; moreover, there is a uniform magnetic field in the $\mathrm{z}$-direction so that the wave equation is given by $-\nabla^{2} u-\frac{2 Z}{r} u+\frac{\epsilon}{i}\left(x u_{y}-y u_{x}\right)=\lambda u, \epsilon$ is a small parameter representing the perturbation and $\lambda$ is related to the energy (it is the eigenvalue of the equation).

Now, if $\epsilon=0$ (no perturbation), the eigenvalues, $\lambda_{n}$, are then given by $\lambda_{n}=-\frac{Z^{2}}{n^{2}}, \quad n=1,2,3, \cdots ;$ and the corresponding eigentates are $u_{n ; i, m}(r, \theta, \varphi)=f_{n ; l}(r) P_{l}^{m}(\cos \theta) \mathrm{e}^{i m \varphi}, \quad l=0,1, \cdots, n-1 ; \quad m=0, \pm 1, \cdots, \pm l . \mathrm{Bu}$, $\frac{1}{i}\left(x u_{y}-y u_{x}\right)=\frac{1}{i} \frac{\partial u}{\partial \varphi}$. The effect of this last operator is clear, since $\frac{1}{i} \frac{\partial u_{n ; i, m}}{\partial \varphi}=m u_{n ; l, m}$. Hence, the eigenvalue, in the presence of perturbation, is given by $\lambda_{n}(\epsilon)=-\frac{Z^{2}}{n^{2}}+\epsilon m$. While the eigenfunctions do not change with the perturbation [6].

Thence, the magnetic field removed the degeneracy in eigenvalues, e.g. if $l=1$ then $m=0, \pm 1$; and the energy levels become $-\frac{Z^{2}}{n^{2}}-\epsilon,-\frac{Z^{2}}{n^{2}},-\frac{Z^{2}}{n^{2}}+\epsilon$, respectively.

\section{Concluding Discussion}

In this brief review article, we have shown the importance of perturbation theory in evaluating the eigenvalues and eigenfunctions for physical systems in many applied fields, in classical mechanics, fluid mechanics, heat flow in certain bodies, and most prominent in quantum mechanics where we gave sample examples of 
well-known problems in the literature and cited in the references given below.

Perturbation theory will keep to be a vital subject leading to many applications in many fields; as an example, recently a $\mathrm{PhD}$ dissertation is written on perturbation theory and its role in the evaluation of Feyman Integrals using integration by parts identities [16].

\section{Conflicts of Interest}

The authors declare no conflicts of interest regarding the publication of this paper.

\section{References}

[1] Awin, A.M. (2010) Numerical Methods. 1st Edition, Misurata University Publishing, Misurata.

[2] Awin, A.M. (2003) Lectures on Mathematical Methods. 1st Edition, Dar Alkitaab Aljadeed, Beirut.

[3] Benmousa, K.A. (2014) The Numerical Solution of Schrodinger Equation. MSc Thesis, University of Tripoli, Tripoli.

[4] Robertson, E. (2019) Perturbation Theory. http://en.wikpedia.org/wiki/perturbationtheory

[5] Jenann, I. (2019) Theory-Quantum Mechanics. http://en.wikpedia.org/wiki/perturbation

[6] Rellich, F. (1969) Perturbation Theory of Eigenvalue Problems. 1st Edition, Gordon and Breach Science Publishers, New York.

[7] Hunter, J. (2017) Asymptotic Analysis and Singular Perturbation Theory. Chapter 1, 1-17. http://www.math.ucdavis.edu

[8] Dushman, S. (2018) Element of the Quantum Theory. https://www.scribd.com/document/378218401/Perturbation-theory

[9] Young, W.R. (2017) Part A: Perturbation Theory. University of California at San Diego, La Jolla, 1-9.

[10] Ziock, K. (1969) Basic Quantum Mechanics. 1st Edition. Willey and Sons, New York.

[11] Rienstra, S. (2018) JMBC Course. https://www.win.tue.nl/ sjoerdr/Q/Onderwijs/JMBC-Cursus-2018.pdf

[12] Van Dyke, M. (1975) Perturbation Methods in Fluid Mechanics. Annotated Edition, Academic Press, New York.

[13] Debbio, L. (2013) Perturbation Theory. https://www2.ph.ed.ac.uk/ ldeldebb/docs/QM/lect17.pdf

[14] Kerley, G.I. (2008) New Developments in Time-Independent Quantum-Mechanical Perturbation Theory. https://arxiv.org/ftp/arxiv/papers/1306/1306.6912.pdf

[15] Bransden, B.H. and Joachain, C.J. (2000) Quantum Mechanics. 2nd Edition, Pearcon Education Ltd., Essex.

[16] Georgoudis, A. (2019) Topics in Perturbation Theory. PhD Thesis, Acta Universitatis, Upsaliensis. 